\title{
Black-body luminance and magnitudes per square arcsecond in the Johnson-Cousins BVR photometric bands
}

\author{
Salvador Bará \\ Dept. Física Aplicada, Universidade de Santiago de Compostela, 15782 Santiago de Compostela, Galicia
}

Received August 15, 2019; accepted September 20, 2019; published September 30, 2019

\begin{abstract}
A relevant number of light pollution studies deal with unwanted visual effects of artificial light at night, including the anthropogenic luminance of the sky that hinders the observation of celestial bodies which are the main target of ground-based astrophysical research, and a key asset of the intangible heritage of humankind. Most quantitative measurements and numerical models, however, evaluate the anthropogenic sky radiance in any of the standard Johnson-Cousins UBVRI photometric bands, generally in the V one. Since the JohnsonCousins V band is not identical with the visual $\operatorname{CIE~} V(\lambda)$ used to assess luminance, the conversion between these two photometric systems turns out to be spectrum-dependent. Given its interest for practical applications, in this Letter we provide the framework to perform this conversion and the transformation constants for black-body spectra of different absolute temperatures.
\end{abstract}

One of the side-effects of the use of artificial light at night is the increase of night sky brightness above its expected natural levels [1], due to the scattering of upward light emissions off the molecular and aerosol constituents of the atmosphere [2]. The calculation and measurement of the integrated spectral sky radiance are very often made in the widely used astrophysical Johnson-Cousins $\mathrm{V}$ photometric band [3], and its visual effects, in terms of luminance, are loosely identified with the ones that would be obtained if the correct visual spectral sensitivity band $V(\lambda)[4]$ were used instead.

However, since the Johnson-Cousins (JC) V and the CIE $\mathrm{V}(\lambda)$ bands are not coincident, the relationship between the integrated radiance in both of them turns out to be dependent on the spectrum of the incident light [5-6]. The same applies to the blue (B) and red (R) JC bands (Fig. 1). Given the widespread use of JC $\mathrm{V}$ band integrated radiances to deduce approximate estimates of visual percepts, the purpose of this Letter is to provide an accurate framework for the transformation between these two systems, and its application to black-body spectra of different absolute temperature. Explicit constants for the transformations from $\mathrm{B}$ and $\mathrm{R}$ bands integrated radiances are also provided in graphical form. The $\mathrm{U}$ (ultraviolet) and I (near infrared) bands of the UBVRI JC photometric system are of lesser interest for this particular application and will not be included here, although the corresponding transformation constants can be straightforwardly obtained following the steps outlined below.

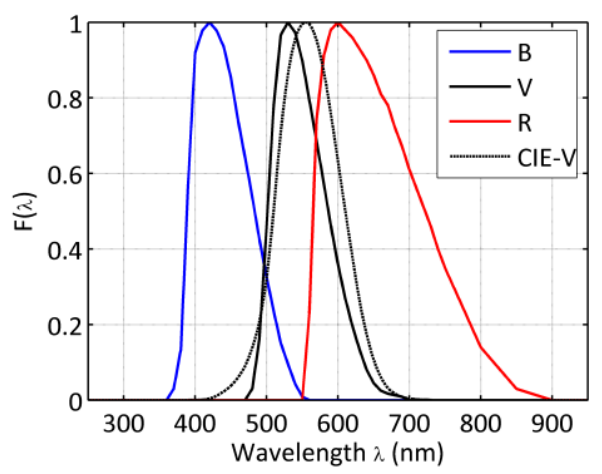

Fig. 1. CIE $V(\lambda)$ and Johnson-Cousins B, V, and R bands.

Let us remind that the luminance $L$ (units $\mathrm{cd} / \mathrm{m}^{2}$ ) produced by a spectral radiance distribution $L_{r}(\lambda)\left(\mathrm{W} \cdot \mathrm{m}^{-2} \mathrm{sr}^{-1} \cdot \mathrm{nm}^{-1}\right)$ for photopically adapted observers is obtained by weighting it by the $\operatorname{CIE~} \mathrm{V}(\lambda)$ function and integrating across wavelengths:

$$
L=k_{m} \int V(\lambda) L_{r}(\lambda) \mathrm{d} \lambda,
$$

where $k_{m}=683 \mathrm{~lm} / \mathrm{W}$ is the scaling factor relating luminance to radiance for monochromatic radiation at wavelength $\lambda_{0}=555 \mathrm{~nm}$, corresponding to the maximum of the $\mathrm{V}(\lambda)$ function.

The night sky brightness corresponding to the same $L_{r}(\lambda)$ spectral radiance, in turn, is customarily expressed in astrophysics in the non-SI, negative logarithmic scale of units called "magnitudes per square arcsecond", which for any generic JC filter band, $F(\lambda)$, with $F \in\{B, V, R\}$ are defined as:

$$
m_{F}=-2.5 \log _{10} \frac{\int F(\lambda) L_{r}(\lambda) \mathrm{d} \lambda}{\int F(\lambda) L_{r 0}(\lambda) \mathrm{d} \lambda},
$$

where $L_{r 0}(\lambda)$ is the arbitrary, but clearly specified, spectral radiance distribution that is chosen to set the origin of the magnitudes scale.

From Eqs. (1) and (2) one immediately obtains:

$$
L=L_{0} \times 10^{-0.4 m_{F}},
$$

where the "zero-point" radiance $L_{0}\left(\mathrm{~cd} / \mathrm{m}^{2}\right)$ is given by 


$$
L_{0}=k_{m}\left\{\frac{\int V(\lambda) L_{r}(\lambda) \mathrm{d} \lambda}{\int F(\lambda) L_{r}(\lambda) \mathrm{d} \lambda} \int F(\lambda) L_{r 0}(\lambda) \mathrm{d} \lambda\right\rceil
$$

Note that an important and frequently overlooked factor for the correct interpretation of the meaning of $m_{T}$ and of the conversion Eqs. (3) and (4), is the particular choice of radiance distribution taken as reference, $L_{r 0}(\lambda)$. The scale of astronomical magnitudes - henceforth denoted by $\mu_{T}$ and not to be confounded with the magnitudes per square arcsecond, $m_{T}$, see below- corresponds to band integrated irradiances $E\left(\mathrm{~W} \cdot \mathrm{m}^{-2}\right)$ and its zero point has been defined differently across history, using e.g. sunbased or Vega ( $\alpha$ Lyr) spectral irradiance distributions [7]. A more convenient choice for our present purposes, not tied to the experimental determination of the spectral irradiance produced by any particular star, is the absolute (AB) magnitude scale, whose reference spectral irradiance $E_{r 0}(v)$ is constant when expressed in the frequency $(\mathrm{Hz})$ domain, and is implicitly defined by [8]:

$$
\mu_{A B}=-2.5 \log _{10} E_{r}(v)-48.60,
$$

with the measured irradiances $E_{r}(v)$ expressed in ergs $\cdot \mathrm{s}^{-1} \cdot \mathrm{cm}^{-2} \cdot \mathrm{Hz}^{-1}$. Note that in Ref. [9] this equation has a misprint in the sign of the -48.60 zero-point term. From Eq. (4), the $\mathrm{AB}$ zero-point reference spectral irradiance distribution in the frequency domain $E_{r 0}(v)$ (i.e., $E_{r}(v)$ for $\left.\mu_{A B}=0\right)$ is, with four significant digits,

$$
E_{r 0}(v)=10^{-0.4 \times 48.60}=3631 \times 10^{-20} \mathrm{ergs}^{\cdot} \mathrm{s}^{-1} \cdot \mathrm{cm}^{-2} \cdot \mathrm{Hz}^{-1},
$$

or, equivalently, $E_{r 0}(v)=3631 \mathrm{Jy}$, where $1 \mathrm{Jy}$ (jansky) = $10^{-26} \mathrm{~W} \cdot \mathrm{m}^{-2} \cdot \mathrm{Hz}^{-1}$. The spectral irradiance in the frequency domain can be expressed in the wavelength domain taking into account that $v=\mathrm{c} / \lambda$, where $\mathrm{c}=2.99792458 \times 10^{8} \mathrm{~m} \cdot \mathrm{s}^{-1}$ is the speed of light, and that $E_{r 0}(v) \mathrm{d} v=E_{r 0}(\lambda) \mathrm{d} \lambda$. Since $|\mathrm{d} v / \mathrm{d} \lambda|=\mathrm{c} / \lambda^{2}$, it follows that $E_{r 0}(\lambda)=3631 \times 10^{-26} \times \mathrm{c} / \lambda^{2} \mathrm{~W} \cdot \mathrm{m}^{-2} \cdot \mathrm{m}^{-1}$, with $\mathrm{c}$ expressed in $\mathrm{m} \cdot \mathrm{s}^{-1}$ and $\lambda$ in $\mathrm{m}$.

The corresponding zero-point radiance, $L_{r 0}(\lambda)$, is defined as the spectral radiance that would give rise to the zero-point irradiance, $E_{r 0}(\lambda)$, under normal incidence, if the source would subtend a solid angle $\Delta \omega=1$ square arcsecond as seen from the observer. Since $1 \operatorname{arcsec}^{2}=$ $2.3504 \times 10^{-11} \mathrm{sr}$, we finally have, for the $\mathrm{AB}$ zero-point spectral radiance, in $\mathrm{W} \cdot \mathrm{m}^{-2} \cdot \mathrm{sr}^{-1} \cdot \mathrm{m}^{-1}$,

$$
L_{r 0}(\lambda)=\frac{E_{r 0}(\lambda)}{\Delta \omega}=\frac{3631 \times 10^{-26}}{2.3504 \times 10^{-11}} \times \frac{c\left[\mathrm{~m} \mathrm{~s}^{-1}\right]}{\lambda^{2}[\mathrm{~m}]} .
$$

With this $\mathrm{AB}$ reference radiance we can calculate the zero-point luminance in Eq. (4) for any particular spectral radiance distribution whose JC magnitudes per square arcsecond are given by $m_{F}$ in Eq. (3). A particular subset of spectra of practical interest are the black-body ones. Their spectral radiance is given by

$$
L_{r}(\lambda)=\frac{2 h c^{2}}{\lambda^{5}} \times \frac{1}{\exp \left(\frac{h c}{\lambda k T}\right)-1},
$$

where $T$ is the absolute temperature (in kelvin, $\mathrm{K}$ ), $h=6.62607015 \times 10^{-34} \mathrm{~J} \cdot \mathrm{s}$ is the Planck constant, and $k=1.380649 \times 10^{-23} \mathrm{~J} \cdot \mathrm{K}^{-1}$ is the Boltzmann constant, according to the 2019 redefinition of SI base units.

The results for $L_{0}\left(\mathrm{~cd} / \mathrm{m}^{2}\right)$ of the $\mathrm{B}, \mathrm{V}$ and $\mathrm{R}$ bands are displayed in Fig. 2. The curve corresponding to the $\mathrm{V}$ band, of particular practical interest, is shown in Fig. 3.

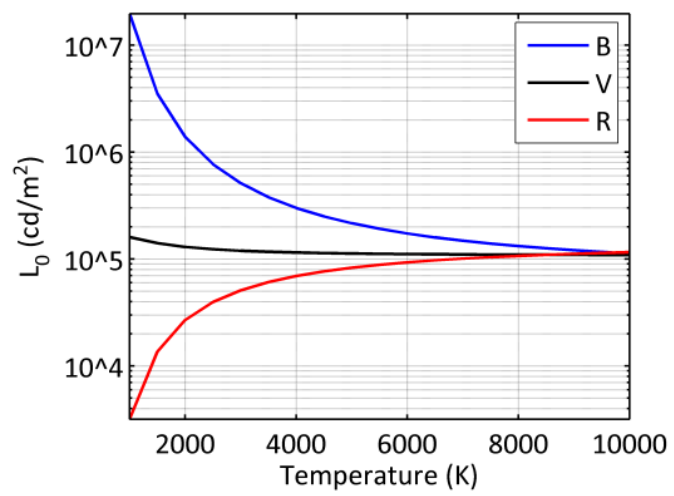

Fig. 2. Zero-point luminances for black-body source radiation of different temperatures, corresponding to the $\mathrm{AB}$ magnitude per square arcsecond system for the Johnson-Cousins B, V, and R bands.

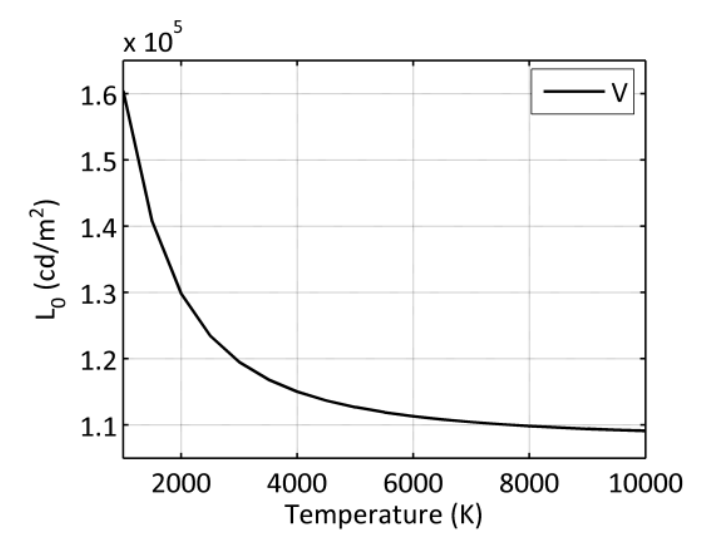

Fig. 3. Detailed view of the zero-point luminances for black-body source radiation of different temperatures, corresponding to the $\mathrm{AB}$ magnitude per square arcsecond system for the Johnson-Cousins V band.

Given the widespread use of the JC V-magnitudes to estimate luminances, the numerical values of $L_{0}$ at $\mathrm{T}$ intervals of $500 \mathrm{~K}$ are specified in the second column of Table 1. 
Table 1. Zero-point luminances $L_{0}$ for black-body source radiation of different temperatures, corresponding to the $\mathrm{AB}$ magnitude per square arcsecond system for the Johnson-Cousins V band, and modified zeropoint luminances $L_{0}{ }^{\prime}$ for a Johnson-Cousins $\mathrm{V}$ system in which the magnitude of Vega ( $\alpha$ Lyr) be identically equal to 0.00 .

\begin{tabular}{ccc}
\hline $\begin{array}{c}\text { Black body } \\
\text { temperature }\end{array}$ & $\begin{array}{c}\boldsymbol{L}_{\boldsymbol{o}} \\
\text { (in units } \\
\mathbf{1} \text { (K) }\end{array}$ & $\begin{array}{c}\mathbf{L}^{\mathbf{5}} \mathbf{c d}^{\mathbf{}} \mathbf{m}^{\mathbf{2}} \text { ) } \\
\text { (in units } \\
\mathbf{1 0}^{\mathbf{5}} \mathbf{c d}^{\mathbf{m}} \mathbf{m}^{\mathbf{2}} \text { ) }\end{array}$ \\
\hline 1000 & 1.6044 & 1.5751 \\
1500 & 1.4071 & 1.3814 \\
2000 & 1.2979 & 1.2742 \\
2500 & 1.2345 & 1.2120 \\
3000 & 1.1949 & 1.1731 \\
3500 & 1.1686 & 1.1473 \\
4000 & 1.1502 & 1.1292 \\
4500 & 1.1369 & 1.1161 \\
5000 & 1.1269 & 1.1063 \\
5500 & 1.1192 & 1.0987 \\
6000 & 1.1131 & 1.0928 \\
6500 & 1.1083 & 1.0881 \\
7000 & 1.1044 & 1.0842 \\
7500 & 1.1012 & 1.0811 \\
8000 & 1.0985 & 1.0784 \\
8500 & 1.0962 & 1.0762 \\
9000 & 1.0943 & 1.0743 \\
9500 & 1.0926 & 1.0727 \\
10000 & 1.0912 & 1.0712 \\
\hline
\end{tabular}

The values of $L_{0}$ for the $\mathrm{V}$ band in Fig. 3 and Table 1 can be directly used to calculate the luminance $L$ of the black-body radiation whose $\mathrm{AB} \mathrm{V}$-band magnitudes per square arcsecond are $m_{V, A B}$, as

$$
L=L_{0} \times 10^{-0.4 m_{V, A B}},
$$

see Eq. (3).

As a final remark, if instead of the $\mathrm{AB}$ spectral radiance given in Eq. (6) the spectral radiance of the star Vega ( $\alpha$ Lyr) were taken as the reference for setting the zero-point of the V-magnitude scale, the magnitudes per square arcsecond in both systems would be related by [10]

$$
m_{V, A B}-m_{V, \text { Vega }}=0.02
$$

Hence, using Vega as the reference, the luminances of the black-body sources in terms of $\mathrm{V}$-magnitudes per square arcsecond are given by

$$
L=L_{0} \times 10^{-0.4 \times 0.02} \times 10^{-0.4 m_{V, V e g a}}=L_{0}{ }^{\prime} \times 10^{-0.4 m_{V, V e g a}} .
$$

The values of the zero-point luminances in this shifted magnitude scale, $L_{0}{ }^{\prime}$, are given in the third column of Table 1.

The above results show that the most commonly used transformation between $\mathrm{V}$-magnitudes per square arcsecond and luminances, which is based on a generic zero-point luminance of $1.08 \times 10^{-5} \mathrm{~cd} / \mathrm{m}^{2}$ has not universal validity, even if restricted to the measurement of radiation from black-body sources. For this last type of spectra, especially for moderate to low color temperatures, the values provided in Table 1 should be used instead.

This work was supported by Xunta de Galicia/FEDER, grant ED431B 2017/64.

\section{References}

[1] F. Falchi et al., Sci. Adv. 2, e1600377 (2016). doi:10.1126/sciadv. 1600377

[2] M. Kocifaj, J. Quantitative Spectroscopy Radiative Transfer 181, 2 (2016).

[3] M.S. Bessel, Publications of the Astronomical Society of the Pacific, 102, 1181 (1990).

[4] CIE, Commision Internationale de l'Éclairage. CIE $19882^{\circ}$ Spectral Luminous Efficiency Function for Photopic Vision (Vienna, Bureau Central de la CIE, 1990).

[5] S. Bará, Intern. J. Sustainable Lighting IJSL 19(2), 104 (2017). doi: 10.26607/ijsl.v19i2.77

[6] A. Sánchez de Miguel, M. Aubé, J. Zamorano, M. Kocifaj, J. Roby, C. Tapia. Monthly Notices of the Royal Astronomical Society 467(3), 2966 (2017). doi:10.1093/mnras/stx145

[7] M.S. Bessell, Annual Reviews of Astronomy and Astrophysics, 43, 293 (2005).

[8] J.B. Oke, The Astrophysical J. Suppl. Series 236(27), 21 (1974).

[9] J.B. Oke, J.E. Gunn, The Astrophysical J. 266, 713 (1983).

[10] M.R. Blanton, S. Roweis S., The Astronomical J. 133(2), 734(2007). 